Rev. Saúde públ., S. Paulo

$9: 417-25,1975$

\title{
ATUALIZAÇŐES/CURRENT COMMENTS
}

\section{IDENTIFICAÇÃO E AVALIAÇÃO DOS FATORES CLINICOS DA GESTAÇÃO DE ALTO RISCO *}

\author{
Peđro Augusto Marcondes de Almeida** \\ Cyro Ciari Junior ** \\ Arnaldo Augusto Franco de Siqueira ** \\ Néia Schor ** \\ Sueli Gandolfi Giron ** \\ Ana Cristina d'Andretta Tanaka**
}

RSPU-B/281

\begin{abstract}
Almeida, P. A. M. et al. - Identificação e avaliação dos fatores clínicos da gestação de alto risco. Rev. Saúde públ., S. Paulo, 9:417-25, 1975.

Resumo: $E$ de grande importancia atual, a identificação das gestantes de alto risco, no sentido de se poder oferecer uma eficiente assistencia durante o ciclo gravídico puerperal. Nesse sentido objetivou-se facilitar tal identificação, estabelecendo uma tabela dividida em 4 grupos de fatores clínicos, que possam a vir determinar ou agravar a gestação de alto risco.
\end{abstract}

UNitermos: Assistência pré-natal. Risco gravídico.

Risco é uma palavra que indica probabilidade de lesão, dano ou perda; e risco gravídico é definido como sendo a oportunidade a agravos físicos, psíqui$\cos$ e sociais a que estão expostos a gestante e o feto. A gestação acarreta condições especiais tanto do ponto de vista físico, como psíquico e social, consideradas próprias do estado gravídico.

O Comitê de Assistência Materno-Infantil da Associação Médica Americana define gestação de alto risco como "aquela que tem uma alta probabilidade de apresentar uma criança com impedimento físico, intelectual, social ou de personalidade, que possa dificultar o crescimento e o desenvolvimento normais e a capaci- dade para aprender. Este impedimento pode ser originado no período pré-natal, perinatal ou pós-natal e pode resultar de influências hereditárias ou ambientais desfavoráveis, atuando em separado ou em combinação".

Existe uma série de fatores, que atuando de forma isolada, praticamente nada acarretam em relação ao risco gravídico. Outros por si só, já determinam alto risco. A associação de vários fatores, mesmo de pequena importância, podem levar a uma gestação de alto risco.

0 risco gravídico deve ser encarado sob dois aspectos: o risco materno e o fetal. Por suas características, os fatores que elevam o risco materno sempre de-

* Trabalho apresentado no XI Congresso Brasileiro de Ginecologia e Obstetricia, realizado de 31 de agosto a 6 de setembro de 1975, Rio de Janeiro.

* Da Disciplina Higlene Materna do Departamento de Prática Médica em Saúde Pública da Faculdade de Saúde Pública da USP. 
ALMEIDA, P. A. M. de et al, - Identificação e avaliação dos fatores clínicos da gestação de alto risco. Rev. Saúde públ., S. Paulo, 9:417-25, 1975.

terminam correspondente elevação do risco fetal, mas este pode agravar-se sem afetar o risco materno.

Além do mais, devemos lembrar que o risco gravídico tem seu aspecto clínico, social e de comportamento da gestante. Este trabalho visa estabelecer parâmetros do ponto de vista clínico para a identificação da população gestante que necessita de uma maior atenção de pessoal especializado e de exploração técnica mais aprofundada.

$\mathrm{Na}$ avaliação destes parâmetros enfrentamos alguns problemas; assim, a observação clínica pode falhar e é variável de acordo com a interpretação do obstetra. Por outro lado, os fatores que, efetivamente, atuam acarretando gestação de alto risco, são difíceis de serem analisados quantitativamente, quer sob o ponto de vista individual ou quando se associam vários fatores.

Nesbitt e Aubry ${ }^{5}$, em 1969, estabeleceram, após estudos desde 1960, um índice de assistência sanitária materno-infantil julgando ser o juízo clínico insuficiente para a identificação deste índice, porque depende de muitos aspectos além de fatores objetivos de risco. Estes autores agruparam 8 fatores ou variáveis, quantificando cada um deles com uma contagem arbitrária de acordo com a presença de circunstâncias desvantajosas para a gestante. A soma da pontuação total dos oito grupos menos 100 fornece-nos o índice de assistência. $O$ resultado igual a 70 ou menos indica gestação de alto risco.

Vários trabalhos realizados no Departamento de Ginecologia e Obstetrícia da Universidade do Estado de Nova York mostraram falha deste índice, primeiro porque foi aplicado a um grupo de gestantes que eram reconhecidamente de alto risco e em segundo lugar não ocorreu variação de pontos durante toda a gestação até o parto.

Em um empenho adicional os autores aplicaram um índice de trabalho de parto com a finalidade de diferenciar as pacientes de alto risco das de baixo risco.

Goodwin e col. ${ }^{3}$, em 1969, idealizaram outro tipo de pontuação que incluía fatores identificáveis no início da gestação, outros identificáveis em etapas mais tardias e os que apareciam no pré-parto e no parto. Observaram que nenhuma morte perinatal ocorreu em gestante com pontuação baixa, o mesmo acontecendo em relação a recém-nascidos com depressão grave. Relatam, também, que quanto maior a pontuação, mais baixa a concentração de estriol urinário.

Não há dúvida de que podemos identificar a população obstétrica de alto risco e que deste grupo originar-se-á a maior parte da mortalidade e morbilidade perinatal. $\mathrm{O}$ mesmo aspecto observamos para - lado das complicações maternas.

Marmol e col. ${ }^{4}$ observaram que cerca de $70 \%$ das mortes maternas ocorreram em mulheres que foram identificadas como de alto risco. Ciari e Almeida ${ }^{2}$, estudando retrospectivamente óbitos maternos no município de Osasco e identificando vários indicadores na história destas gestantes, concluíram que se às mesmas tivesse sido aplicado algum índice, elas seriam catalogadas como de alto risco.

Através disto, podemos, identificando esta população, estabelecer certo enfoque para a centralização de pessoal, fundos de assistência e aplicação de cuidados técnicos e de conhecimento mais refinados.

A aplicação destes índices no início da gestação permite identificar, na experiência de Aubry e Pennington ${ }^{1}, 66 \%$ da população de alto risco, nesta época da gestação. Só $33 \%$ é incluída durante a evolução da gestação, em épocas próximas do trabalho de parto e no parto. Outro aspecto importante é que, segundo os autores, só $20 \%$ dos recém-nascidos que apresentam problemas, surgem do grupo de gestantes de baixo risco.

Portanto, as pacientes identificadas como de alto risco ingressariam em um sis- 
ALMEIDA, P. A. M. de et al. - Identificacão e avaliação dos fatores clínicos da gestação de alto risco. Rev. Saúde públ., S. Paulo, 9:417-25, 1975.

tema de assistência perinatal intensiva refletindo uma colaboração entre obstetras e neonatologistas.

Tentamos estabelecer uma tabela de índice que leva em conta vários aspectos clínicos e dividimos este índice em 4 grupos que incluam determinados tipos de fatores.

No primeiro grupo reunimos uma série de fatores de alto risco, aplicáveis na primeira consulta pré-natal ou em qualquer outra fase da gestação na qual se identifique um destes fatores. Este grupo identificaria sempre gestantes de alto risco. Quando ao aplicarmos este grupo de fatores à gestante não identificamos nenhum deles, ele é considerado 0 e o risco dependerá do comportamento dos demais fatores dos outros três grupos. Se for diferente de 0 , isto é, se a gestante apresentar um determinado fator que a inclua neste grupo, apresentará índice 1 definindo seu alto risco permanente que é agravado ou melhorado pelo comportamento, dos demais fatores dos outros grupos, e relacionado com recursos assistenciais disponiveis. Os fatores incluídos neste primeiro grupo e que identificam alto risco permanente, só deixarão de ser aplicados quando forem devidos a afecções passiveis de correção cirúrgica, durante a gestação (Anexo I).

Um segundo grupo seriam os fatores pessoais e familiares de risco clínico, aplicáveis somente na primeira consulta. Estes fatores são identificados através de determinada pontuação negativa que assumirá valores diferentes de acordo com a significação de cada variável. A soma da pontuação total fornece-nos o grau de risco. Assim tomamos um escore de 100 pontos e consideramos até 70 baixo risco, de 65 a 50 médio risco e 45 e menos alto risco (Anexo II).

No terceiro grupo reunimos os fatores evolutivos aplicáveis durante o evolver do controle pré-natal. Estes fatores também são identificados com pontuação negativa que mudará de acordo com o significado de cada variável. $\quad 0$ escore obtido seguirá a mesma orientação do $2 .^{\circ}$ grupo.

Este terceiro grupo permitirá identificar gestantes de alto risco como também avaliar a evolução do risco em gestantes que já foram assim identificadas, após a aplicação dos fatores do primeiro ou do segundo grupo (Anexo III).

0 quarto grupo incluirá fatores aplicáveis às gestantes, que realizando o prénatal, já estejam identificadas pelos 3 grupos anteriores como de alto, médio ou baixo risco, mas que apresentem intercorrências agudas clínicas, cirúrgicas ou obstétricas, identificáveis somente durante o trabalho de parto. Estas variáveis não necessitam ser medidas como as dos grupo II ou III por serem sempre determinantes de alto risco (Anexo IV).

Deveremos ressaltar, além do mais, que naquelas gestantes que não realizaram pré-natal ou se o fizeram não foram submetidas à aplicação de nenhuma das tabelas anteriores, os 4 grupos de fatores, anteriormente descritos, poderão ser aplicados desde que se tenha os dados do grupo evolutivo III. Caso isto não aconteça os fatores do grupo I, II e IV poderão, efetivamente, ser aplicados.

Objetivamente, o estudo prospectivo das gestantes às quais for aplicado este índice nos permitirá, através do acompanhamento da grávida e da evolução de seu produto conceptual, determinar: a) o peso de cada um dos fatores que contribuem para um maior ou menor risco; b) identificar quais os fatores que se associam mais freqüentemente ocasionando gestação de alto risco e aumentando a incidência de morbimortalidade materna e neonatal. 0 estudo prospectivo das gestantes, assim identificadas, poderão possibilitar-nos, ainda considerando os recursos disponíveis, enfocar a assistência que deverá ser fornecida a uma determinada população de grávidas, como também implicar em alterações tanto no que diz respeito à quantificação, como também à retirada ou inclusão de outros fatores. 
ALMEIDA, P. A. M. de et al. - Identificação e avaliação dos fatores clínicos da gestação de alto risco. Rev. Saude públ., S. Paulo, 9:417-25, 1975.

\title{
A NEXO I
}

\begin{abstract}
Grupo I - Fatores de alto risco aplicáveis na primeira consulta ou em qualquer época do ciclo gravidico puerperal, definindo sempre como alto risco permanente. Os fatores incluidos neste grupo e que identificam alto risco permanente, só deixarão de sê-lo quando forem afecçóes passiveis de correção cirúrgica durante a gestação e que isto efetivamente ocorra.
\end{abstract}

Cardiopatias classe II-III ou IV

Tuberculose pulmonar ativa

Acidente vascular cerebral anterior Miastenia grave

Corela

Tumor cerebral

Retocolite ulcerativa crônica

Cirrose hepática

Esquistossomose hépato-esplênica com hipertensão porta

Diabetes latente

Diabetes declarado

Hipertiroldismo

Hipotiroldismo

Hiperparatiroidismo

sindrome de Cushing

Doença de Addison

Feocromocitoma

Lupus eritematoso disseminado

Hipertensão arterial por moléstia renal crônica

Sindrome nefrótico

Moléstia renal crônica
Doença hemolitica

Anemia aplásica

Púrpura trombocitopênica idiopática

Leucemia

Doença de Hodgkin

Tumores malignos

$\mathrm{Ca}$ de colo uterino

Classe IV ou $\mathrm{V}$ de Papanicolaou

Abortamentos repetidos em número maior do que 3 (excluindo provocado)

obito fetal intra-uterino habitual em número maior do que 3

obito neonatal repetido em número malor do que 3

Prematuridade habitual em número maior do que 3

Malformações repetidas em número maior do que 2

Isoimunização $\mathrm{Rh}$

Esterilidade primária por mais de 5 anos

Esterilidade secundária por mais de 5 anos

A NEXO II

Grupo II - Fatores pessoais e familiares de risco clínico aplicáveis somente na primeira consulta da gestante ou no parto.

I - Dados pessoais

a) Idade materna

Pontuação

até 15 anos

$-35$

16 a 19 anos

$-10$

20 a 28 anos

29 a 35 anos

0

36 a 40 anos

15
-25

41 ou mais

$-35$

b) Estado marital

casada

solteira, casada, desquitada, viúva (vivendo só)

$-10$

solteira, desquitada, viúva (com união estável)

c) Altura

menor que $1,50 \mathrm{~m}$ 
ALMEIDA, P. A. M. de et al. - Identificação e avaliação dos fatores clínicos da gestação de alto risco. Rev. Saude pribl., S. Paulo, 9:417-25, 1975.

II - História obstétrica

a) Paridade

Primigesta

2 a 4

$-5$

5 ou mais

0

Primigesta idosa (29 anos ou mais) - acrescentar

$-10$

Primigesta jovem (até 15 anos) - acrescentar

$-20$

Primigesta funcional - acrescentar

- 20

$-20$

b) Evolução das gestaçōes anteriores

Normais

Com intercorrências obstétricas anteriores (mola hidatiforme gravidez ectópica - hiperêmese gravídica - toxemia gravídica - descolamento prematuro de placenta - placenta prévia e outras)

Com rotura uterina anterior

c) Partos anteriores

Normais

Fórcipes

Cesárea 1

1
2

2

4 ou mais

Apresentacões viciosas recurrentes

d) Dequitação e puerpério

Dequitação normal

Retenção de placenta

0
$-\quad 5$

Hemorragia após parto *

(se mais de 2)

$-5$

Inversão aguda de útero

$-20$

Puerpério normal

- 5

Infecção puerperal

$-5$

e) Abortos de qualquer tipo

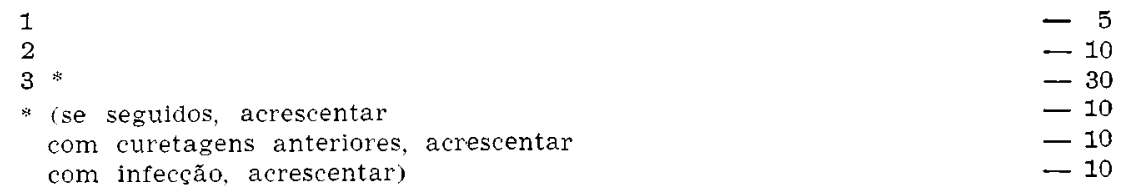

f) Obito fetal intra-útero

$\begin{array}{ll}1 & -10 \\ 2 & \\ 3 * & -20 \\ * \text { (se seguidos, acrescentar) } & -10\end{array}$ 
ALMEIDA, P. A. M. de et al. - Identificação e avaliação dos fatores clinicos da gestação de alto risco. Rev. Saúde puibl., S. Paulo, 9:417-25, 1975.

g) Peso ao nascer

$\begin{array}{lr}\text { Menos de } 2.000 \mathrm{~g} & -20 \\ 2.001 \text { a } 2.500 \mathrm{~g} & -10 \\ 2.501 \text { a } 3.000 \mathrm{~g} & -5 \\ 3.001 \text { a } 4.500 \mathrm{~g} & \\ 4.501 \text { ou mais * } & \\ \text { *se mais de um recém-nascido acima de } 4.000 \mathrm{~g} \text {, acrescentar) } & -10\end{array}$

h) Nascimentos prematuros (menos de 37 semanas)

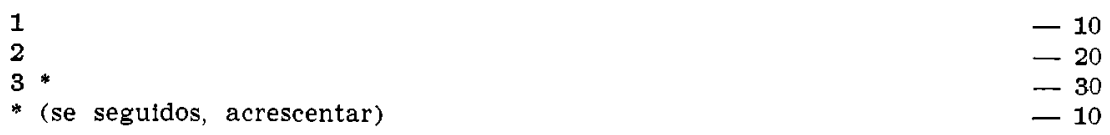

1) Problemas com recém-nascido: malformações, anomalias genéticas ou de causa indeterminada

$\begin{array}{ll}1 & -20 \\ 2 & -30 \\ 3 & -55\end{array}$

III - Doenças associadas à gravidez

Cardiopatias Classe I

Tuberculose pulmonar inativa *

* (se operada, acrescentar) - 10

Asma brônquica

Epilepsia

Pré-diabetes (história familiar - ou história pessoal de fetos
gigantes ou de óbitos fetais sem diagnóstico)

Obesidade

Hipertensão arterial cardiovascular $\quad-35$

Doenças psiquiátricas $\quad-10 \mathrm{a}-20$

Qualquer outra moléstia, segundo critério médico, de acordo com o dano sobre mãe ou feto

IV - Ginecopatias

Esterilidade primária 1 a 2 anos

2 a 5 anos
Esterilidade secundária 2 a 5 anos

$-10$

$-35$

História de cirurgia ginecológica anterior

Conização do colo uterino

Amputação de colo uterino

Outras cirurgias por via baixa

Cirurgias incidindo sobre o corpo uterino

Mioma uterino (dependendo da localização)

Anomalias uterinas congênitas (dependendo do tipo)

Outras de acordo com o dano sobre mãe ou feto

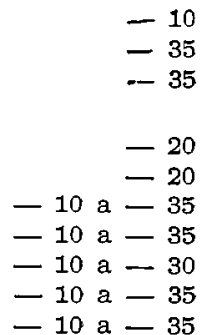

Avaliação do risco

Tomando-se um escore de 100 pontos consideramos

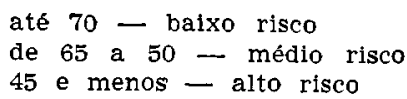


ALMEIDA, P. A. M. de et al. - Identificação e avaliação dos fatores clínicos da gestação de alto risco. Rev. Saúde puibl., S. Paulo, 9:417-25, 1975.

\section{A NEXO III}

Grupo III - Fatores evolutivos aplicáveis no controle da evolução do pré-natal. Servirá para identificar gestantes de alto risco, como também avaliar a evolucão do risco em gestantes que já foram identificadas como de baixo, médio ou alto risco após aplicação dos fatores do grupo I ou do grupo II.

- Sindromes hemorrágicas

1. trimestre - Aborto evitável se único

Pontuasão

Se em gestantes com 2 ou mais abortos anteriores $\quad-5 \overline{5}$

$-10$

Prenhez ectópica -10

Mola hidatiforme

3.0 trimestre - Placenta prévia

$-55$

Descolamento prematuro de placenta $\quad$ - 55

- Vômitos simples

$-10$

- Vômito grave

$-20$

- Gestação gemelar

- Polihidramnio

- Estado de mal asmático

- Estado de mal epilético

- Edema agudo de pulmão

- Acidente vascular cerebral na gestação

- Hematêmese ou melena

- Coma diabético

- Crise tireotóxica

- Processos homorrágicos por doenças hematológicas

$-35$

$-35$

- Anemia 10 a 11

$$
9 \text { a } 10 \mathrm{~g}
$$

$-35$

$-35$

$-55$

$-55$

$-55$

- Bacteriúria assintomática

- Infecção urin

$-55$

- 55

$-55$

$-5$

$-10$

$-35$

$-10$

$-40$

- Glomerulonefrite aguda

- Sífilis - tratada antes do $4 .{ }^{\circ}$ més

$-55$

tratada após o $4 .^{\circ}$ mês

não tratada

$-10$

$-55$

- Cancro mole

$-10$

- Blenorragia

$-10$

- Granuloma inguinal

$-10$

- Linfogranuloma venéreo

$-10$

- Toxoplasmose aguda

- Hepatite

- Rubéola - $1.0^{\circ}$ mês

$-55$

$-35$

$20^{\circ}$ mês

$-55$

$30^{\circ}$ mês

após 3. ${ }^{\circ}$ mês

$-55$

$-35$

$-10$

- Outras viroses até o $3 .^{\circ}$ mês

$-35$

- Outras viroses após o $3 . \circ$ mès

$-10$

Complicaçōes cirúrgicas (apendicite aguda - pancreatite, úlcera gastro-

duodenal perfurada - obstrucão intestinal aguda e outras)
Qualquer outra intercorrência segundo critério médico, de acordo com o dano sobre mãe ou feto

Dados do controle pré-natal

P.A. Aumento da pressão sistólica de $30 \mathrm{~mm} \mathrm{Hg}$ ou da pressão diastólica de $15 \mathrm{~mm} \mathrm{Hg}$ 
ALMEIDA, P. A. M. de et al. - Identificação e avaliação dos fatores clínicos da gestação de alto risco. Rev. Saúde públ., S. Paulo, 9:417-25, 1975.

Ganho de peso após o primeiro trimestre

1 a $1,5 \mathrm{~kg}$ por mês

+ de $1,5 \mathrm{~kg}$ por mês

0
-35

de 0,5 a $1 \mathrm{~kg}$ por mês

$-20$

menos de $0,5 \mathrm{~kg}$ por mês

$-35$

(dos 2 últimos itens exclui-se a obesa)

Edema

$-35$

Altura uterina $4 \mathrm{~cm} / \mathrm{mês}$

mais de $4 \mathrm{~cm}$ (excluindo gemelar-polihidramnio)

0

se associado a estados diabéticos acrescentar

$-10$ menos de $4 \mathrm{~cm}$

se associado a alguma intercorrência clinica (hipertensão aterial

$-35$

por exemplo), acrescentar

Suspeita de óbito fetal intra-útero

$-10$

obito fetal intra-útero

$-35$

Situação transversa após 37.a semana

$-55$

Apresentação pélvica após 37: semana

$-35$

Fundo de olho $A_{0} \mathrm{H}_{1}$

qualquer outro resultado diferente do $\mathrm{A}_{0} \mathrm{H}_{0}$

$-35$

Estriol urinário ou plasmático

curva de evolução apresentando 2 quedas sucessivas em níveis decrescentes

Lactogênio placentário

curva de evolução apresentando 2 quedas sucessivas em niveis decrescentes

Amniocentese - liquido meconial

Amnioscopia - liquido meconial

$-55$

Prova de esforço (Stembera) positiva

$-55$

Proteinúria presente

Glicosúria presente

Dados de comportamento
1 ausência a consulta ou a exames laboratoriais agendados
$-10$
2 ausências a consulta ou a exames laboratoriais agendados
$-35$
3 ausências a consulta ou a exames laboratoriais agendados
$-55$

Temor excessivo, atitudes hostis, gestações anteriores não controladas, época tardia de inscrição

\section{Avaliação do risco}

Tomando-se o escore de 100 pontos consideramos

$$
\begin{aligned}
& \text { até } 70 \text { - baixo risco } \\
& \text { de } 65 \text { a } 50 \text { - médio risco } \\
& 45 \text { e menos - alto risco }
\end{aligned}
$$

Todas as condições previstas neste grupo e que acarretem determinado tipo de risco, delxam de ser consideradas caso sejam corrigidas com alguma conduta médica: clinica, obstétrica ou cirúrgica. Tal fato poderá acarretar diminuição do risco ou até desaparecimento total do mesmo. 
ALMEIDA, P. A. M. de et al. - Identificação e avaliação dos fatores clínicos da gestação de alto risco. Rev. Saúde públ., S. Paulo, 9:417-25, 1975.

\title{
A NEXO IV
}

\begin{abstract}
Grupo IV - Fatores de parto: aplicáveis às gestantes que fizerem pré-natal e que apresentem por ocasião da internação em qualquer época da gestação ou durante o trabalho de parto, alguma intercorrência obstétrica. Elas não serão quantificadas, pois sempre são encaradas como de alto risco.
\end{abstract}

Mola hidatiforme

Abortamento infectado

Perfuração uterina após aborto provocado

Toxemia aguda pré-eclâmpsia ou eclâmpsia

Placenta prévia

Descolamento prematuro de placenta

Rotura uterina

Inversão aguda de útero

Hemorragia traumática (colo, fundos de saco vaginal, vulva e perineo)

Atonia uterina

Retencão de placenta

Placenta acreta

Embolia amniocaseosa

Coaguiopatia
Insuficiência renal aguda

Amniorrexis prematura

Infecção intra-parto

Procúbito de cordão umbilical

Prolapso de cordão umbilical

Vasa prévia

Liquido amniótico meconial (Amnioscopia Amnlocentese - Rotura artificial ou espontânea de bolsa)

Variação dos batimentos cardíacos fetals

óbito fetal intra-uterino

Transversa abandonada

Apresentação pélvica

Distócia funcional

Desproporção céfalo-pélvica

Tumor prévio

RSPU-B/281

Almeida, P. A. M. de et al. - [Identification and evaluation of clinical factors in high-risk pregnancies]. Rev. Saúde públ., S. Paulo, 9:417-25, 1975.

SUMMARY: The identification of high-risk pregnancies is very important, because it allows correct protection of pregnancy and puerperium. In order to simplify this procedure, a risk chart containing four groups of clinical factors which might determine or aggravate high-risk pregnancies, is presented.

UNITERMS: Prenatal care. Pregnancy, risk.

\section{REFERENCIAS BIBLIOGRAFICAS}

1. AUBRY, R. H. \& PENNINGTON, J. C. Identification and evaluation of highrisk pregnaniv. The perinatal concept. Clin. Ubstet. and Gynec., 16: 3-27, 1973.

2. CIARI Jr., C. \& ALMEIDA, P. A. M. de - Elementos de avaliação do risco gravídico. Rev. Saúde públ., S. Paulo, 6:57-78, 1972.

3. GOODWIN, J. W. et al. - Antepartum identification of the fetus at risk. Can. med. Assoc. J., 101:458-64, 1969.
4. MARMOL, J. G. et al. - Maternal death and high-risk pregnancy: an analysis of 40 maternal deaths in the collaborative project. Obstet, and $G y$ nec., 30:816-20, 1967.

5. NESBITT Jr., R. E. L. \& AUBRY, R. H. - High risk obstetrics. II. Value or semiobjetive grading system in identifying the vulnerable group. Amer. J. Obstet. Gynecol, 103:972-85, 1969.

Recebido para publicaç̃o em 06-06-75 Aprovado para publicacão em 30-06-75 